\title{
Voice Recognition and Voice Navigation for Blind using GPS
}

\author{
Manisha Bansode $^{1}$, Shivani Jadhav ${ }^{2}$, Anjali Kashyap ${ }^{3}$ \\ Assistant Professor, Electronics, SPIT, Mumbai, India ${ }^{1}$ \\ Student, Electronics, SPIT, Mumbai, India ${ }^{2,3}$
}

\begin{abstract}
In this paper we have presented the design of the voice based navigation system for blind using voice recognition module and GPS module implemented on arduino board. This system helps the blind person to navigate. The blind person will give the destination's name as the input to voice recognition module. GPS module continously receives the latitude and longitude of the current location. GPS compares it with the destination's latitude and longitude. The blind person receives the pronounced directions which he needs to follow to reach his destination.
\end{abstract}

Keywords: Arduino, GPS, Navigation, Voice Recognition

\section{INTRODUCTION}

India is now home to the world's largest number of blind people. Of the 37 million people across the globe who are blind, over 15 million are from India. There has been many efforts but even now, it is not easy for blind people to independently move. At present several devices are available for providing guidance to a remote location but these are either expensive or not easy to use.

In order to overcome daily challenges, the traditional method used by a visually impaired person are walking cane and guide dog but this methods are ineffective as it is not reliable. With the rapid advances in modern technology, both in hardware and software have brought potential to provide smart aid for impaired people.

Advances in wearable computing, voice recognition, wireless communication, GIS and GPS have made possible to address the visually impaired and disabled navigation problem.

Electronic Travel Aids designed and devised to help the blind people to navigate safely and independently. Several devices available at present for providing guidance to a remote location are either expensive or difficult to handle.

The system explained here provides a cost effective and reliable solution to the above problem. The main objective of our project is to design a small and simple navigation device to help the blind people to get navigation directions through audio message which depends on the real-time assistance pro-vide by the Global Positioning System.

GPS is the acronym for Global Positioning System. It is employed to find the position where the user is located on the earth. This information is provided by the GPS receiver with the help of the data it receives from the satellites.

Voice recognition technology is the process of identifying, understanding and converting voice signals into text or commands.

\section{BLOCK DIAGRAM}

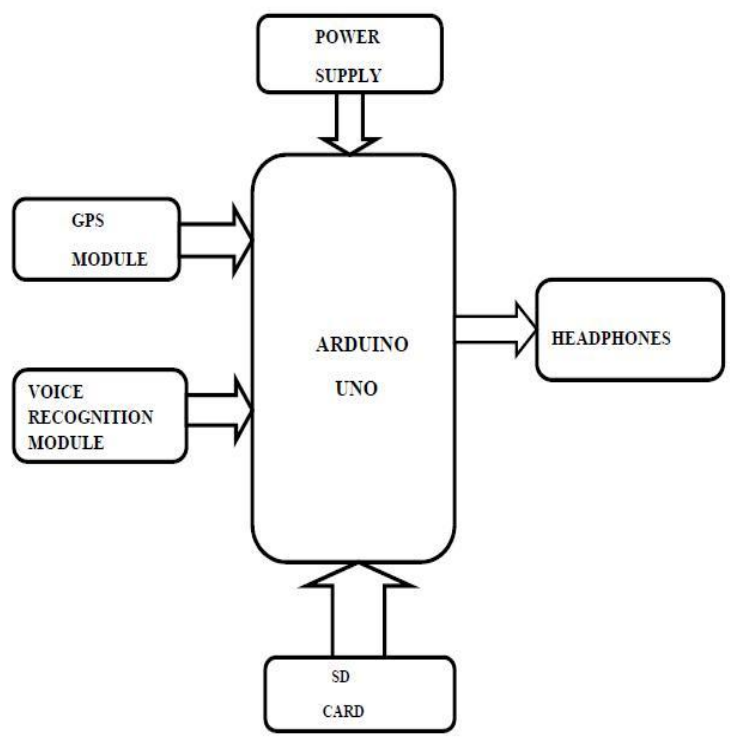

Fig. 1: Block Diagram

The block diagram of our project is as shown in fig. 1 ,which consists of GPS receiver, arduino, Voice recognition module ,Headset, SD card reader, SD card and other supplementary components.

The blind person gives the destination's name as the input to the voice recognition module. The GPS continuosly receives the latitude and longitude of the current location.

The system compares the destination with the stored locations in the database and selects the latitude and longitude of the destination location. With the help of the code uploaded in the arduino the GPS gives the navigation directions .

These navigation directions are stored in the sd card in audio format. So the blind person will then hear the directions stored in sd card with the help of head phones. 


\section{HARDWARE DESCRIPTION}

\section{A. ARDUINO}

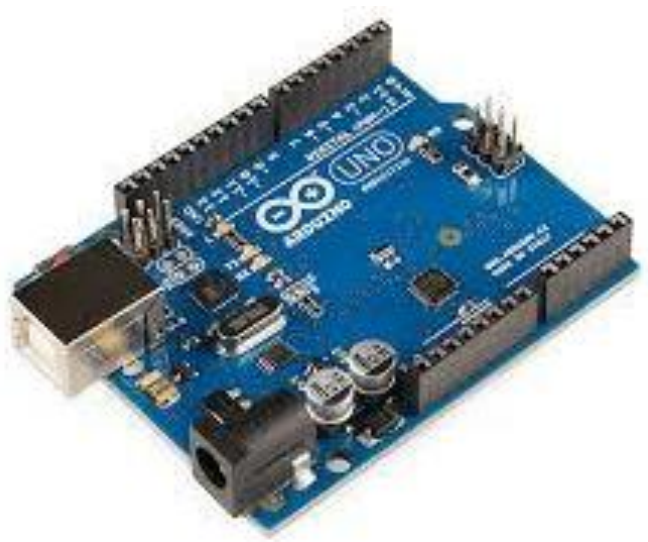

Fig. 2: Arduino

Arduino is an open-source computer hardware and software company, project and user community that designs and manu-factures kits for building digital devices and interactive objects that can sense and control the physical world. An Arduino board consists of an Atmel 8bit AVR microcontroller with complementary components that facilitate programming and incorporation into other circuits. The Arduino integrated de-velopment environment (IDE) is a cross-platform application written in Java, and derives from the IDE for the Processing programming language and the Wiring projects. It includes a code editor with features such as syntax highlighting, brace matching, and automatic indentation, and is also capable of compiling and uploading programs to the board with a single click. A program or code written for Arduino is called a sketch.

Arduino programs are written in $\mathrm{C}$ or $\mathrm{C}++$. The Arduino IDE comes with a software library called "Wiring" from the original Wiring project, which makes many common input/output operations much easier. Users only need define two functions to make a runnable cyclic executive program. Features:

1) ATmega 328 microcontroller

2) Input voltage - 7-12V

3) 14 Digital I/O Pins (6 PWM outputs)

4) 6 Analog Inputs

5) 32k Flash Memory

6) $16 \mathrm{Mhz}$ Clock Speed

\section{B. GPS}

The Global Positioning System (GPS) is a satellite-based navigation system made up of a network of 24 satellites placed into orbit. A GPS receiver must be locked on to the signal of at least three satellites to calculate a $2 \mathrm{D}$ position (latitude and longitude) and track movement.

With four or more satellites in view, the receiver can determine the user's 3D position (latitude, longitude and altitude). Once the user's position has been determined, the GPS unit can calculate other information, such as speed, bearing, track, trip distance, distance to destination, sunrise and sunset time and more.

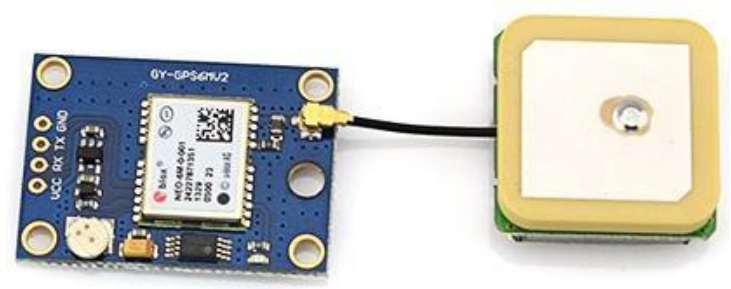

Fig. 3: GPS module neo6mv2

We have used Ublox NEO6MV2 GPS Module. Some of the features of this module is listed below:

1. Power Supply Range: $3 \mathrm{~V}$ to $5 \mathrm{~V}$; Model: GYGPS6MV2

2. Ceramic antenna; EEPROM for saving the configuration data when powered off

3. Backup battery; LED signal indicator; Mounting Hole Diameter: $3 \mathrm{~mm}$

4. Default Baud Rate: 9600 bps; Module size 23mm * $30 \mathrm{~mm}$

5. Antenna size $12 * 12 \mathrm{~mm}$; Cable: $20 \mathrm{~mm}$

\section{VOICE RECOGNITION MODULE}

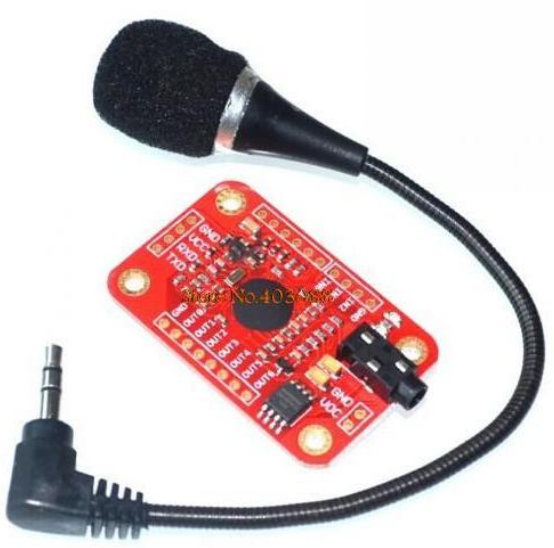

Fig. 4: Voice Recognition Module V3

The blind person gives destination's name as the input to the voice recognition module. The system compares the destination with the stored locations in the database. We have used Voice Recognition Module V3. Voice Recognition Module is a compact and easy-control speaking recognition board. This product is a speakerdependent voice recognition module. It supports up to 80 voice commands in all. Max 7 voice commands could work at the same time. Any sound could be trained as command. Users need to train the module first before let it recognizing any voice command. This board has 2 controlling ways: Serial Port (full function), General Input Pins (part of function). General Output Pins on the board could generate several kinds of waves while corresponding voice command was recognized. Some of the parameters of $\mathrm{V} 3$ are are listed below:

1. Voltage: $4.5-5.5 \mathrm{~V}$

2. Current: $40 \mathrm{~mA}$

3. Digital Interface: $5 \mathrm{~V}$ TTL level for UART interface and GPIO 
4. Analog Interface: $3.5 \mathrm{~mm}$ mono-channel microphone connector and microphone pin interface

5. Size: $31 \mathrm{~mm} \times 50 \mathrm{~mm}$

6. Recognition accuracy: 99

D. SD Card module

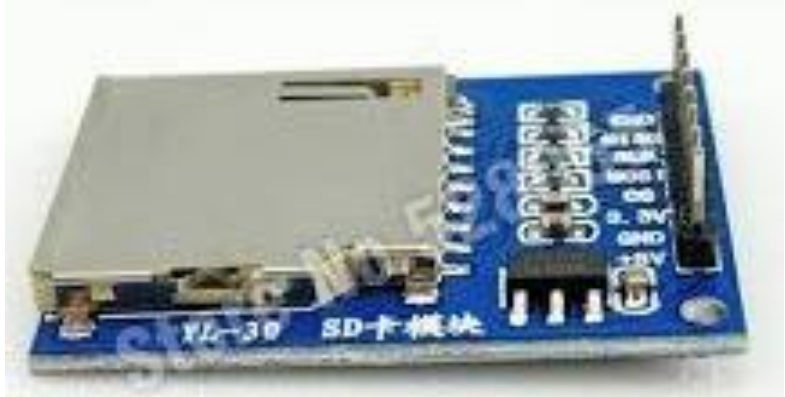

Fig. 5: SD Card module

It allows to read and write to the SD card using your arduino through programming.It is easily interfaced as a peripheral to your arduino sensor shield module.It can be used for SD Card more easily, such as for MP3 Player, MCU/ARM system control

With all SD SPI Pins out: MOSI, SCK, MISO and CS ,for further connection Input Voltage: $3.3 \mathrm{~V} / 5 \mathrm{~V}$

The navigation directions are stored in audio format in the SD Card with the help of which the blind person gets the directions.

IV. SOFTWARE DESCRIPTION

In order to implement various functionalities we have used various libraries of arduino.

\section{A. TinyGPS++}

TinyGPS++ is designed to provide most of the NMEA GPS functionality.It gives an Arduino user - position, date, time, altitude, speed and course - without the large size that seems to accompany similar bodies of code. To keep resource consumption low, the library avoids any mandatory floating point dependency and ignores all but a few key GPS fields. It transforms the raw NMEA latitude and longitude val-ues into floating-point numbers representing decimal degrees. TinyGPS++ makes it easy to check speed, time, date, and course

\section{B. SimpleSDAudio}

Play audio files with your Arduino in decent quality from SD card, only very few additional hardware required. Features-

1. 8-Bit PWM output - no external DAC required

2. $\quad 62.500 \mathrm{kHz}$ (fullrate) / $31.250 \mathrm{kHz}$ (halfrate) sampling rate@16 MHz

3. $\quad 31.250 \mathrm{kHz}$ (fullrate) / $15.625 \mathrm{kHz}$ (halfrate) sampling rate@8 Mhz

4. Mono, bridge and stereo mode

5. RAM usage $1.3 \mathrm{kByte}$

6. ROM usage $6.1 \mathrm{kByte}$

7. Integrated SD library (minimal FAT only, optimized for low RAM usage and high performance)

8. Works with most SD card shields that are hooked to SPI port

9. Easy to use API: 1. init library, 2. select audio file, 3. call play

10. Supports SD and SDHC cards formated with FAT16 or FAT32

C. Voice Recognition V3

It is responsible for all the functionalities of the voice recognition module. It is because of this library that the Voice Recognition V3 module stores voice and executes the required program.

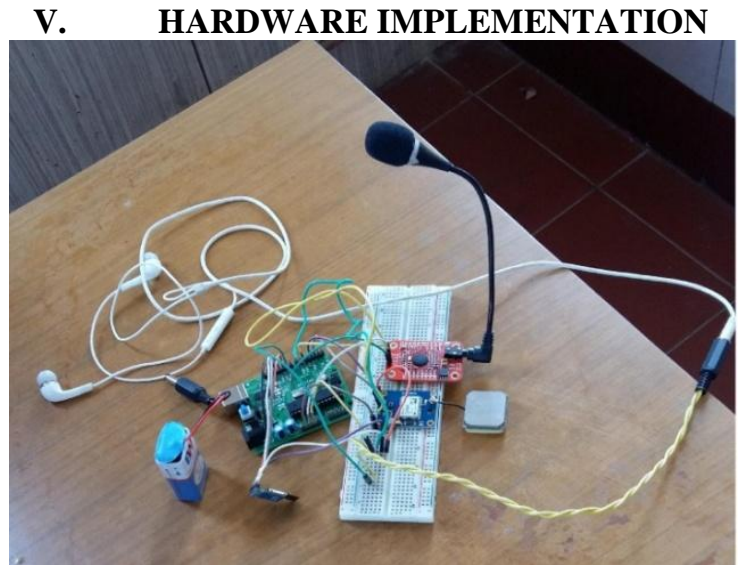

Fig. 6: Hardware implementation

\section{RESULT}

We have tested our system where our current location was Saradr Patel Institute of Technology and destination was Bhavans gate. We gave the destination's name as the input to the voice recognition, the program takes the corresponding latitude and longitude. The GPS starts giving the distance between the the two locations, direction to destination, current speed etc as shown in the fig.6. In our actual implementation instead of printing the directions, the directions will be heard by the blind person.

We have stored the latitude and longitude of the destination in the program. In the above case the latitude was 19.124719 and longitude was 72.836761 i.e. latitude and longitude of Bhavans Gate.

We got the distance between the two locations that was initially 120.49 metres.We also got the current speed of the user. As the user moves the distance between the two location changes, speed of the user changes and subsequently the user gets the instruction. All these have been displayed in Fig.6.

In our final implementation the user gives the destination as voice input to the voice recognition module which automati-cally takes the destination's latitude and longitude.

The instruc-tions were converted into audio files which were stored in SD card and the user hears the instructions through headphones. 
INTERNATIONAL JOURNAL OF INNOVATIVE RESEARCH IN ELECTRICAL, ELECTRONICS, INSTRUMENTATION AND CONTROL ENGINEERING Vol. 3, Issue 4, April 2015

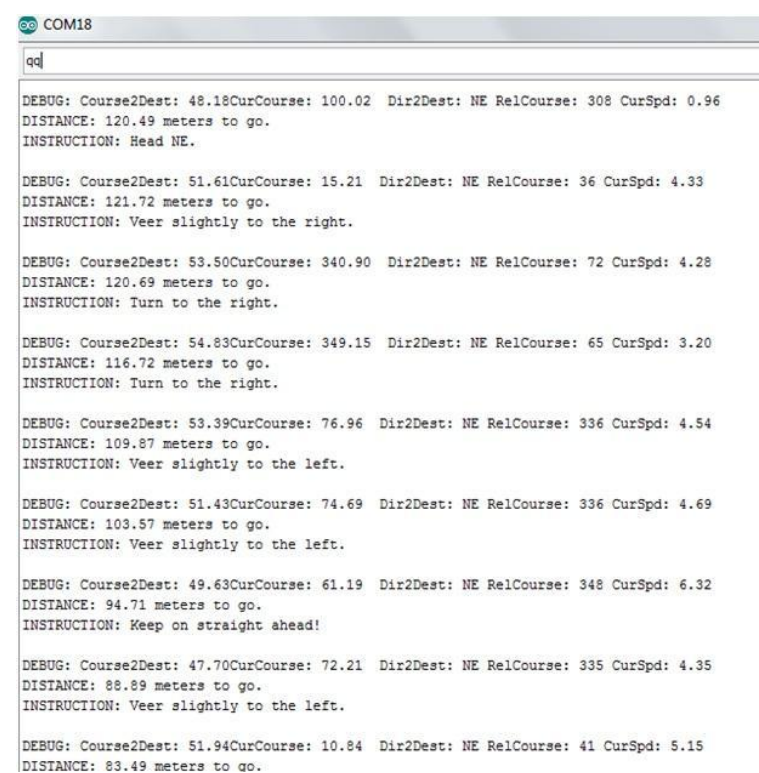

Fig. 7: System giving directions from S.P.I.T to Bhavans Gate

\section{CONCLUSION}

India is one of the countries with world's largest population of blind people. Initially many blind people prefer to not use electronic aids, and use only canes or guide dogs. This is because of relatively high costs and poor levels of user satisfaction associated with existing electronic systems. So we tried to develop a low cost and user friendly system for blind people. This method offers innovative solutions in order to replace the conventional methods of guiding blind person. The system designed consists of a GPS receiver and a voice recognition module which is interfaced to the Arduino. Arduino is programmed in such a way that depending on the satellite information about the destination, the blind person gets the navigation directions. We navigate the blind people using voice.

However, there are some limitations of our system. We cannot use it indoors because no signals can be received from the GPS module. Further, the users often need to learn for a period of time to trust the system and more evaluation of the system in the real situation is required. Therefore, we have to work on to improve user trust in terms of its accuracy of perceiving environmental information

During designing of the device we faced some problems such as:

1. We tried to use TMRpcm library for playing the audio files but have to drop that idea as it is interrupt driven.

2. This system is not suitable for indoor building or closed area, so we test the device outdoor only.

3. The RAM of Arduino is relative small. So we decreased the buffer size and transferred most of the statement in flash memory.

4. This system takes time to start because of GPS. As GPS takes time to receive data from the satellites.

5. This system can be used for only limited number of places.

\section{FUTURE SCOPE}

This project can be extended by incorporating a GSM module. We can interface this module to send messages to the near and dear ones of the Blind person regarding his/her current position. Doing so, we can track the movement of the Blind person in a very efficient manner.

We can also incorporate ethernet shied to this circuit by which the user can go to any place using google maps.

\section{ACKNOWLEDGMENT}

The authors are thankful to the Sardar Patel Institute of Technology, India for providing the necessary facilities for carrying out this work.

Lastly we are thankful to our colleagues and friends for their continuous feedback and suggestion.

\section{REFERENCES}

[1] Harsha Gawari,"Voice and GPS Based Navigation Sys-tem For Visually Impaired",Int. Journal of Engineer-ing Research and Applications ISSN : 2248-9622, April 2014,Vol. 4, Issue 4( Version 6).

[2] Ameer H. Morad "'GPS Talking to Blind People", JOUR-NAL OF EMERGING TECHNOLOGIES IN WEB IN-TELLIGENCE, AUGUST 2010, VOL.2, NO.3

[3] Somnath koley, Ravi Mishra, "VOICE OPERATED OUT-DOOR NAVIGATION SYSTEM FOR VISUALLY IM-PAIRED PERSONS", International Journal of Engineering Trends and Technology-2012, Volume 3, Issue 2

[4] Nandish M S, Mr. Chetan Balaji, Prof. Shantala C P, "An Outdoor Navigation With Voice Recognition Security Application For Visually Impaired People", International Journal of Engineering Trends and Technology (IJETT), Apr 2014- Volume 10 Number

[5] Jae Sung Cha, Dong Kyun Lim, Yong-Nyuo Shin, "Design and Implementation of a Voice Based Navigation for Visually Impaired Persons", International Journal of Bio-Science and Bio-Technology Vol.5,No.3,June, 2013

[6] Rishabh Gulati, "GPS Based Voice Alert System for the Blind", International Journal of Scientific Engineering Research, Volume 2, Issue 1, January-2011 ISSN 2229-5518 